\title{
SUR LES RONGEURS
}

\section{HOTES NATURELS DE SCHISTOSOMA RODHAINI BRUMPT}

\author{
Par J. STIJNS
}

Suite à la redécouverte de $S c h$. rodhaini par le Prof. J. Schwetz, lors de sa courte mission à Elisabethville en 1950, nous avons procédé à la recherche des hôtes naturels de ce schistosome, aussi bien chez les humains que tout spécialement chez les divers rongeurs. Et nous dirons en passant que la recherche chez les rongeurs nous avait été suggérée par le regretté Professeur E. Brumpt (suggestion transmise par le $\mathrm{D}^{\mathrm{r}}$ Schwetz). Voici le résultat de nos recherches.

La recherche systématique des œufs de $S c h$. rodhaini dans les excréta et dans le produit de râclage de la muqueuse intestinale chez des animaux sauvages capturés dans la région d'Elisabethville a permis de connaître les hòtes naturels de ce parasite. Tous appartiennent à l'ordre des rongeurs, sous-ordre des Simplicidentés, famille des Muridés; ce sont :

Lophuromys aquilus, True.

Praomys jacksoni, de Winton.

Pelomys frater, Thomas.

Mastomys coucha, Smith.

Cinq spécimens du Muridé Tatera nyassæ ont été négatifs. Il en a été de même de neuf spécimens de Crocidura luna Dollman, insectivore ubiquiste, dont l'éthologie ressemble à celle de Mastomys ; chez un de ces Crocidura, l'examen du produit de râclage de la muqueuse intestinale a montré la présence d'œufs de Sch. mansoni * . Des œufs de $S c h$. rodhaini n'ont jamais été trouvés dans le produit de râclage de la muqueuse intestinale chez. des batraciens, oiseaux et reptiles capturés à proximité des gîtes à cercaires de $S c h$. rodhaini. Enfin, il convient de signaler que des œufs de Sch. rodhaini n'ont jamais été trouvés dans les selles d'une vingtaine de travail-

(1) Constatation à confirmer par des recherches ultérieures. (N.D.L.R.).

Ann. de Parasitologie, T. XXVII, $\mathrm{N}^{\circ} 4 .-1952$. 
leurs indigènes préposés au curage des ruisseaux et des canaux infectés de planorbes à cercaires de $S c h$. rodhaini; par contre, ces travailleurs étaient fortement parasités par Sch. mansoni.

Il semble donc très probable que $S c h$. rodhaini est une espèce ne parasitant que des rongeurs.

Chez les Muridés naturellement infestés, il n'est pas fréquent de trouver des œufs de $S c h$. rodhaini dans les crottes, même en recourant aux diverses méthodes d'enrichissement. A l'autopsie, on ne trouve généralement qu'un petit nombre de Sch. rodhaini dans le mésentère et dans le foie, en contraste avec l'infestation massive que l'on peut provoquer expérimentalement chez la souris blanche. Les œufs se trouvent dans la partie terminale de l'intestin grêle, le cæcum et le gros intestin. La digestion peptique du foie permet aussi de récolter des œufs, plus ou moins altérés, au point qu'il est quelquefois difficile de reconnaître l'aspect typique des œufs de Sch. rodhaini ; à l'examen histologique, on trouve des granulomes bilharziens dans le parenchyme hépatique.

Les Muridés, hôtes naturels de Sch. rodhaini, fréquentent les bords des rivières et des ruisseaux, sauf Mastomys qui est ubiquiste.

Le plus fréquemment observé sur les berges humides est Lophuromys aquilus. Ce rongeur, qui se rencontre sur tous les versants herbeux des petites vallées de la région d'Elisabethville, semble tolérer un sol très humide ou même marécageux; il vit dans les endroits non couverts, souvent en bordure des galeries, à l'intérieur desquelles il ne s'attarde jamais longuement.

Praomys jacksoni, excellent grimpeur, vit uniquement dans les endroits ombragés, loin ou près de l'eau ; il abonde dans les galeries forestières des environs d'Elisabethville.

Pelomys frater vit toujours à proximité de l'eau, mais rarement près des galeries ; son habitat typique est un terrain oú poussent des herbes très hautes et ensoleillé. Il a été capturé à proximité de nombreuses rivières dans la région d'Elisabethville.

Mastomys coucha se rencontre (en dehors des locaux habités) en habitats dégagés ou ombragés, loin ou près de l'eau, peu fréquemment toutefois dans les galeries forestières denses. Il a été capturé dans tous les endroits piégés dans la région d'Elisabethville, y compris dans la cité européenne.

Nous tenons beaucoup à remercier pour leur assistance technique : MM. Lips, Van Den Bulcke et Peel, et tout spécialement M. Paul Pirlot, qui a bien voulu diriger les piégeages et identifier les rongeurs capturés. 